SU-ITP-92-30

$\mathrm{UG}-10 / 92$

hep-th@xxx/9212030

June 14, 2021

\title{
SUPERSYMMETRIC STRING WAVES
}

\author{
E. A. Bergshoeff 凹 \\ Institute for Theoretical Physics, University of Groningen \\ Nijenborgh 4, 9747 AG Groningen, The Netherlands \\ R. Kallosh [ and T. Ortín [ \\ Physics Department, Stanford University, Stanford CA 94305, USA
}

\begin{abstract}
We present plane-wave-type solutions of the lowest order superstring effective action which have unbroken space-time supersymmetries. They describe dilaton, axion and gauge fields in a stringy generalization of the Brinkmann metric. Some conspiracy between the metric and the axion field is required.

We show that there exists a special class of these solutions, for which $\alpha^{\prime}$ stringy corrections to the effective on-shell action, to the equations of motion (and therefore to the solutions themselves), and to the supersymmetry transformations vanish. We call these solutions supersymmetric string waves (SSW).
\end{abstract}

\footnotetext{
${ }^{1}$ Bitnet: bergshoeff@hgrrug5

${ }^{2}$ On leave of absence from: Lebedev Physical Institute, Moscow. E-mail: kallosh@physics.stanford.edu

${ }^{3}$ Bitnet: tomaso@slacvm
} 


\section{Introduction}

In recent years an active field of research has been the search for and study of non-perturbative solutions to the classical equations of motion of superstring effective field theories and the corresponding sigma-models. Many bosonic solutions of such theories have been discovered. Some special solutions turned out to have a highly non-trivial property: although bosonic, they have some unbroken supersymmetries. This means that when embedded into the right supersymmetric theory they admit Killing spinors.

Bosonic solutions with unbroken supersymmetries in theories of quantum gravity are very special because they have some kind of supersymmetric non-renormalization property. They provide an example of a nontrivial gravitational background in which quantum (super)gravity corrections to the effective on-shell action and (sometimes) even to equations of motion vanish. Until very recently, the only example of a background where all perturbative quantum gravity corrections to the effective action vanish was given by the empty Minkowski space. This suggests that bosonic solutions with unbroken supersymmetries may play an important role in the development of quantum gravity and may help us to understand the structure of the vacuum state in supersymmetric theories.

Arguments that explain these non-renormalization properties were given for supersymmetric string solitons [1] and for extremal black holes [2]. Another particularly interesting kind of metric is provided by the so called $p p$-wavest.

The purpose of this paper is to find metrics in this class which, together with appropriate dilaton, axion and gauge fields, provide solutions of the lowest order superstring effective action and have unbroken supersymmetries. Then we will study how this property and the solutions themselves are affected by stringy corrections in $\alpha^{\prime}[3]$.

There exists an extensive literature on this subject, and we start by introducing $p p$-wave space-times and reviewing some results relevant for our purposes in sec.2.

In sec. 3 we present a general class of solutions to the lowest order $d=10$ heterotic string effective action consisting of a pp-wave metric, dilaton, axion and Yang-MIlls fields. We also show that half of their supersymmetries are unbroken.

In sec. 4 the stringy $\alpha^{\prime}$ corrections will be studied. The metric-axion conspiracy, which was necessary for classical supersymmetry, will be shown to be sufficient to ensure the absence of $\alpha^{\prime}$ corrections to supersymmetry transformations and to the on-shell effective action. However, to get no corrections to the equations of motion one has to constrain the solutions even more by embedding the torsionful spin connection in the gauge group. We call such exact solutions supersymmetric string waves (SSW). Finally, in sec.5 we will present our conclusions.

Appendix A presents a lemma which is used in sec.4 in the proof of the absence of $\alpha^{\prime}$ corrections and Appendix B contains our notation and conventions.

\footnotetext{
${ }^{4}$ Here $p p$-waves stands for plane fronted waves with parallel rays.
} 


\section{Gravitational plane-fronted waves}

Pp-wave geometries are space-times admitting a covariantly constant null vector field

$$
\nabla_{\mu} l_{\nu}=0, \quad l^{\nu} l_{\nu}=0 .
$$

Spacetimes with this property were first discovered by Brinkmann in 1923 [4]. In four dimensions the metrics of these spaces can be written in the general form

$$
d s^{2}=2 d u d v+K(u, \xi, \bar{\xi}) d u^{2}-d \xi d \bar{\xi},
$$

where $u$ and $v$ are light-cone coordinates defined by

$$
l_{\mu}=\partial_{\mu} u, \quad l^{\mu} \partial_{\mu} v=1,
$$

(thus the metric does not depend on $v$ ) and $\xi=x+i y$ and $\bar{\xi}=x-i y$ are complex transverse coordinates. These metrics are classified and described in detail in [5]. Different pp-wave spaces are characterized by different choices of the function $K$ in eq. (2). For example, when $K$ is quadratic in $\xi$ and $\bar{\xi}$,

$$
K(u, \xi, \bar{\xi})=f(u) \xi^{2}+\bar{f}(u) \bar{\xi}^{2}+g(u) \xi \bar{\xi} .
$$

they are called exact plane waves. Plane waves (2) with $K$ of the form

$$
K(u, \xi, \bar{\xi})=\delta(u) f(\xi, \bar{\xi}) .
$$

are called shock waves. A specific example of shock waves is given by the Aichelburg-Sexl geometry [6]

$$
K(u, \xi, \bar{\xi})=\delta(u) \ln (\xi \bar{\xi}) .
$$

which describes the gravitational field of a point-like particle boosted to the speed of light.

Güven established in 1987 [7] that a solution to the lowest order superstring effective action consisting of a straightforward generalization of the four dimensional exact plane waves to $d=10$, dilaton, axion and Yang-Mills fields has half of the $N=1, d=10$ supersymmetries unbroken and is also a solution of the equations of motion of the superstring effective action including all the $\alpha^{\prime}$-corrections. We will describe Güven's solution in detail below. Investigations on the supersymmetry of plane-fronted waves in general relativity were made even earlier. In [8] there is a reference to unpublished work of J. Richer who found that pp-waves are supersymmetric. Very general classes of pp-waves were found by Tod to be supersymmetric in the context of $d=4, N=2$ ungauged supergravity in 1983 [9]. Different aspects of plane wave solutions in string theory have been investigated in the last few years [10]-[12].

The existence of a covariantly constant null vector field has dramatic consequences. For instance, for the class of d-dimensional pp-waves with metrics of the form

$$
d s^{2}=2 d u d v+K\left(u, x^{i}\right) d u^{2}-d x^{i} d x^{i},
$$

where $i, j=1,2, \ldots, d-2$, the Riemann curvature is [12

$$
R_{\mu \nu \rho \sigma}=-2 l_{[\mu}\left(\partial_{\nu]} \partial_{[\rho} K\right) l_{\sigma]} .
$$


The curvature is orthogonal to $l_{\mu}$ in all its indices. This fact is of crucial importance in establishing that all higher order in $\alpha^{\prime}$ terms in the equations of motion are zero due to the vanishing of all the possible contractions of curvature tensors. Güven added dilaton $\phi(u)$, axion $H_{u i j}=b_{i j}(u)$ and Yang-Mills fields $F_{u i}(u)$ which were functions of $u$ only, to a metric of the form eq. (7), choosing $K\left(u, x^{i}\right)$ quadratic in $x^{i}$ (exact plane waves), and then proved the absence of quantum $\left(\alpha^{\prime}\right)$ corrections to the equations of motion [7]. In addition, Güven showed that these solutions have half of the possible supersymmetries unbroken. Moreover he proved that the corrections to the supersymmetry equations vanish. This shows how special these solutions are, especially considering that at the time when Güven did his research, not much was known about $\alpha^{\prime}$ corrections.

Note that supersymmetry played no role in establishing this non-renormalization theorem. Similar results have been obtained in refs. [10], [11], [12].

In arbitrary dimension $d$ the most general metrics admitting a covariantly constant null vector (1), were discovered by Brinkmann in 1925 [13]:

$$
d s^{2}=2 d u d v+A_{\mu}\left(u, x^{i}\right) d x^{\mu} d u-g_{i j}\left(u, x^{i}\right) d x^{i} d x^{j}, \quad l^{\mu} A_{\mu}=0
$$

where $\mu, \nu=0,1, \ldots, d-1$ and $i, j=1,2, \ldots, d-2$. The supersymmetry properties of this general metric have not yet been studied, although they have been discussed recently in the context of string theory and sigma models in [14], [12], [15]. Note that the general Brinkmann metric (9) in $d=10$ has 8 functions $A_{i}\left(u, x^{i}\right)$ and 28 functions $g_{i j}\left(u, x^{i}\right)$ more than the metric (7) investigated by Güven, where only the $u u$-component of the metric $A_{u}=\frac{1}{2} K$ was present and was quadratic in $x^{i}$.

The issue of corrections to the effective string equations of motion for general Brinkmann metrics has been studied by Horowitz [12]. He argued that if the functions $A_{i}$ depend on the coordinates $x^{i}$, the metrics of the solutions do acquire corrections. If, however, this dependence is polynomial, the number of non-vanishing terms that correct the equations of motion is finite for these solutions. An analogous statement was made for the corrections to the axion field: no corrections for $B_{\mu \nu}$ linear in $x^{i}$ s and a finite number of corrections for polynomial dependence.

We will investigate a sub-class of Brinkmann metrics more general than the one studied by Güven. We will consider (9) with flat transverse space, i.e. $g_{i j}\left(u, x^{i}\right)=\delta_{i j}$. The functions $A_{\mu}$ will be arbitrary functions of $u$ and $x^{i}$. First we will identify the solutions to the zero slope limit of the superstring effective action $(N=1, d=10$ supergravity) coupled to Yang-Mills and those which have unbroken supersymmetries in this limit. We will look for solutions with at most one half of the supersymmetries broken. This means that we will admit only one algebraic constraint, which in our case will be

$$
\gamma^{\lambda} l_{\lambda} \epsilon=\gamma^{u} \epsilon=0
$$




\section{String Plane Waves in Ten-Dimensional Einstein -Yang- Mills Supergravity}

Our starting point is the bosonic part of the action of $N=1, d=10$ supergravity coupled to Yang-Mills [16]:

$$
S=\frac{1}{2} \int d^{10} x \sqrt{-g} e^{-2 \phi}\left(-R+4(\partial \phi)^{2}-\frac{1}{3} H^{2}-\frac{1}{2} \beta \operatorname{tr} F^{2}\right),
$$

where $\phi$ is the dilaton, $F_{\mu \nu}$ is the field-strength of the Yang-Mills field $V_{\mu}: F_{\mu \nu}=2 \partial_{[\mu} V_{\nu]}+\left[V_{\mu}, V_{\nu}\right]$, and $H$ is the field-strength of the axion $B_{\mu \nu}$ which includes the Yang-Mills Chern-Simons form:

$$
H_{\mu \nu \rho}=\partial_{[\mu} B_{\nu \rho]}-3 \beta \operatorname{tr}\left\{V_{[\mu} \partial_{\nu} V_{\rho]}-\frac{1}{3} V_{[\mu} V_{\nu} V_{\rho]}\right\} .
$$

The constant $\beta$ is related to the Yang-Mills coupling constant $g$ by $\beta=1 / g^{2}$. Traces are taken in the adjoint representation.

The equations of motion that follow from (11) are

$$
\begin{aligned}
R_{\mu \nu}-2 \nabla_{\mu} \partial_{\nu} \phi+H_{\mu \lambda \rho} H_{\nu}{ }^{\lambda \rho}+\beta \operatorname{tr} F_{\mu \lambda} F_{\nu}{ }^{\lambda} & =0, \\
R-4 \nabla_{\mu} \partial^{\mu} \phi+4(\partial \phi)^{2}+\frac{1}{3} H^{2}+\frac{1}{2} \beta \operatorname{tr} F^{2} & =0, \\
\nabla_{\lambda}\left(e^{-2 \phi} H^{\lambda \mu \nu}\right) & =0, \\
\nabla_{\lambda}\left(e^{-2 \phi} F^{\lambda \mu}\right)+e^{-2 \phi}\left[V_{\nu}, F^{\nu \mu}\right]+e^{-2 \phi} H^{\mu} F^{\lambda \rho} & =0 .
\end{aligned}
$$

In order to investigate the existence of unbroken supersymmetries for our purely bosonic solutions we only need to consider the bosonic terms in the supersymmetry transformation rules of the fermions. They are given by

$$
\begin{aligned}
\delta \psi_{\mu} & =\left(\partial_{\mu}-\frac{1}{4} \Omega_{+\mu}^{a b} \gamma_{a b}\right) \epsilon \\
\delta \lambda & =\left(\gamma^{\mu} \partial_{\mu} \phi-\frac{1}{6} H_{\mu \nu \rho} \gamma^{\mu \nu \rho}\right) \epsilon \\
\delta \chi & =-\frac{1}{4} F_{\mu \nu} \gamma^{\mu \nu} \epsilon
\end{aligned}
$$

where the torsionful spin connections $\Omega_{ \pm \mu}^{a b}$ are defined by

$$
\Omega_{ \pm \mu}^{a b}=\omega_{\mu}^{a b}(e) \pm H_{\mu}^{a b} .
$$

$\omega_{\mu}^{a b}(e)$ is the torsion-free, metric-compatible spin connection, and so the axion field strength $H$ gives torsion to the spin connections $\Omega_{ \pm \mu}^{a b}$. Our Ansatz for the metric will be Brinkmann's with flat transverse space. In our notations?

$$
g_{\mu \nu}=\eta_{\mu \nu}+2 A_{(\mu} l_{\nu)}, \quad g^{\mu \nu}=\eta^{\mu \nu}-2 A^{(\mu} l^{\nu)}-A^{2} l^{\mu} l^{\nu},
$$

${ }^{5}$ The inverse metric can be rewritten avoiding recursiveness as

$$
g^{\mu \nu}=\eta^{\mu \nu}-2 \eta^{\rho(\mu} \eta^{\nu) \sigma} A_{\rho} l_{\sigma}+\eta^{\rho \sigma} A_{\rho} A_{\sigma} \eta^{\mu \alpha} \eta^{\nu \beta} l_{\alpha} l_{\beta} .
$$


where $A_{\mu}=A_{\mu}\left(u, x^{i}\right)$, and $l_{\mu}$ is the covariantly constant null vector (四). For later purposes, we need to reformulate our Ansatz in terms of zehnbein fields

$$
e_{\mu}^{a}=\delta_{\mu}^{a}+A_{\mu} l^{a}, \quad e_{a}^{\mu}=\delta_{a}^{\mu}-A_{a} l^{\mu} .
$$

Our Ansatz for the axion and Yang-Mills fields is

$$
B_{\mu \nu}=3 B_{[\mu} l_{\nu]}, \quad V_{\mu}=V l_{\mu} .
$$

Finally, we will assume that all the fields are independent of the coordinate $v$ but depend arbitrarily on $u$ and $x^{i}$. We also assume that $A_{v}=B_{v}=0$. In covariant notation, $l^{\mu} \partial_{\mu} \phi=l^{\mu} \partial_{\mu} V=l^{\mu} \partial_{\mu} A_{\nu}=$ $l^{\mu} \partial_{\mu} B_{\nu}=0$ and $l^{\mu} A_{\mu}=l^{\mu} B_{\mu}=0$. Note that the component $B_{u}$ is pure gauge but $A_{u}$ is not.

The Christoffel symbols corresponding to (22), and the spin-connection corresponding to (23) are given by

$$
\begin{array}{rlrl}
\left\{\begin{array}{c}
\rho \\
\mu \nu
\end{array}\right\} & =l_{(\mu} \mathcal{A}_{\nu)}^{\rho}+\partial_{(\mu} A_{\nu)} l^{\rho}, & \mathcal{A}_{\mu \nu} & =\partial_{\mu} A_{\nu}-\partial_{\nu} A_{\mu} \\
\omega_{\mu}^{a b} & =-l^{[a} \mathcal{A}_{\mu}^{b]}+\frac{1}{2} \mathcal{A}^{a b} l_{\mu}, & l^{\mu} \mathcal{A}_{\mu \nu}=0 .
\end{array}
$$

Note that the spin connection only depends on $\mathcal{A}_{\mu \nu}$ but the Christoffel symbols (25) depend also on $\partial_{(\mu} A_{\nu)}$. Then, the curvature tensor is:

$$
R_{\mu \nu}^{a b}(\omega)=-l_{[\mu} \partial_{\nu]} \mathcal{A}^{a b}-l^{[a} \partial^{b]} \mathcal{A}_{\mu \nu}-l_{[\mu} \mathcal{A}_{\nu]}{ }^{\alpha} \mathcal{A}_{\alpha}{ }^{[a} l^{b]}
$$

We also need the curvature with respect to the torsionful spin connection (20). The torsion tensor for our Ansatz is given by

$$
H_{\mu}{ }^{a b}=l^{[a} \mathcal{B}^{b]}{ }_{\mu}+\frac{1}{2} \mathcal{B}^{a b} l_{\mu}, \quad \quad \mathcal{B}_{\mu \nu}=\partial_{\mu} B_{\nu}-\partial_{\nu} B_{\mu}
$$

since the Yang-Mills Chern-Simons form vanishes identically in this case. Therefore, the torsionful spin-connections are

$$
\Omega_{ \pm \mu}^{a b}=-l^{[a}(\mathcal{A} \mp \mathcal{B})^{b]}{ }_{\mu}+\frac{1}{2}(\mathcal{A} \pm \mathcal{B})^{a b} l_{\mu}
$$

and for the corresponding torsionful curvature tensor we find

$$
\begin{aligned}
R_{\mu \nu}{ }^{a b}\left(\Omega_{ \pm}\right)= & -l_{[\mu} \partial_{\nu]}(\mathcal{A} \pm \mathcal{B})^{a b}-l^{[a} \partial^{b]}(\mathcal{A} \mp \mathcal{B})_{\mu \nu}- \\
& -l_{[\mu}(\mathcal{A} \mp \mathcal{B})_{\nu]}{ }^{\alpha}(\mathcal{A} \pm \mathcal{B})_{\alpha}{ }^{[a} l^{b]}
\end{aligned}
$$

The expression for the torsionful curvature satisfies the following interchange identity:

$$
R_{\mu \nu, \rho \sigma}\left(\Omega_{ \pm}\right)=R_{\rho \sigma, \mu \nu}\left(\Omega_{\mp}\right)
$$

We are now ready to investigate the supersymmetry properties of our Ansatz. We want to find the supersymmetry transformations that leave the fermions invariant (i.e. equal to zero), that is, all the non-trivial supersymmetry transformation parameters $\epsilon$ for which the r.h.s of eqs. (17)-(19) 
vanish (the Killing spinors). As we explained in the introduction, we consider only solutions with at most one half of the possible supersymmetries broken. From eqs.(17)-(19) we get respectively

$$
\phi=\phi(u), \quad\left[\partial_{\mu}-\frac{1}{8} l_{\mu}(\mathcal{A}+\mathcal{B})_{i j} \gamma^{i j}\right] \epsilon=0, \quad \gamma^{u} \epsilon=0 .
$$

One half of the supersymmetries are always broken. Also, $\epsilon$ is a function of $u$ only, and this implies that $(\mathcal{A}+\mathcal{B})_{i j} \equiv f_{i j}(u)$ must be a function of $u$ only and

$$
\epsilon(u)=e^{1 / 8\left\{\int^{u}\left(f_{i j}(u) \gamma^{i j}\right)\right\}} \epsilon_{0},
$$

where $\epsilon_{0}$ is a constant spinor which satisfies the algebraic constraint $\gamma^{u} \epsilon_{0}=0$. The integrability condition for the existence of Killing spinors is, of course, satisfied:

$$
R_{\mu \nu}^{a b}\left(\Omega_{+}\right) \gamma_{a b} \epsilon=-l_{[\mu} \partial_{\nu]}(\mathcal{A}+\mathcal{B})^{i j} \gamma_{i j} \epsilon=0 .
$$

Finally, substitution of our Ansatz into the equations of motion, eqs. (13)-(16), leads to

$$
\begin{aligned}
& \triangle K+2 \partial^{i} A_{i}^{\prime}+\frac{1}{2} \mathcal{A}^{i j} \mathcal{A}_{i j}-\frac{1}{2} \mathcal{B}^{i j} \mathcal{B}_{i j}+4 \phi^{\prime \prime}-2 \beta \operatorname{tr} \partial^{i} V \partial_{i} V=0, \\
& \triangle V=\partial_{i} \mathcal{A}^{i j}=\partial_{i} \mathcal{B}^{i j}=0
\end{aligned}
$$

where $K=2 A_{u}$, and $\triangle=\partial_{i} \partial_{i}$ is the flat space Laplacian.

\section{String Corrections}

Until now we have treated the constants $\alpha$ and $\beta$ as independent parameters. The field equations could be solved and half of the supersymmetries preserved without relating these parameters to each other. It is well known, however, that in string theory both $\alpha$ and $\beta$ are related to the Regge slope parameter $\alpha^{\prime}$ (inverse string tension) as follows:

$$
\beta=\frac{1}{15} \alpha^{\prime}, \quad \alpha=2 \alpha^{\prime} .
$$

Thus, in this section, we will treat the classical part of the Yang-Mills action $F^{2}$ as coming from string corrections. Here, our starting point will be the zero slope limit $\left(\alpha^{\prime} \rightarrow 0\right)$ of the bosonic part of the effective action. This is given by

$$
S^{(0)}=\frac{1}{2} \int d^{10} x \sqrt{-g} e^{-2 \phi}\left(-R+4(\partial \phi)^{2}-\frac{1}{3}\left(H^{(0)}\right)^{2}\right),
$$

where

$$
H_{\mu \nu \rho}^{(0)}=\partial_{[\mu} B_{\nu \rho]} .
$$

The superscript in $H^{(0)}$ indicates that no $O\left(\alpha^{\prime}\right)$ corrections of any kind are present. Below we will see that the definition of the axion curvature $H$ contains an infinite series of higher order string corrections. 
In this section we are going to consider one particularly simple choice of the functions $A_{i}$ and $B_{i}$ of our Ansatz that solves the above theory:

$$
A_{i}=-B_{i}, \quad f_{i j}(u)=0 .
$$

The Killing spinor in our coordinates is a constant spinor satisfying $\gamma^{u} \epsilon_{0}=0$. In a recent paper by Tseytlin [15] the solution in which the vector function in the metric is related to the one in the axion was mentioned as the most natural one from the point of view of the sigma model equations. It is therefore very interesting to investigate the string corrections to the following class of supersymmetric plane waves. For convenience we rewrite here the different fields and the equations they must satisfy in order to be solutions of the theory (38):

$$
g_{\mu \nu}=\eta_{\mu \nu}+2 l_{(\mu} A_{\nu)}, \quad B_{\mu \nu}=3 l_{[\mu} A_{\nu]}, \quad V_{\mu}=V l_{\mu},
$$

where $l^{\mu} A_{\mu}=A_{v}=0$. All fields are independent of the $v$ coordinate and, furthermore, $\phi$ is a function of $u$ only. We have already included the Yang-Mills fields in the Ansatz (41) but we will not consider them until we discuss first order corrections. The equations that have to be satisfied are

$$
\triangle K+2 \partial^{i} A_{i}^{\prime}+4 \phi^{\prime \prime}=\triangle V=\triangle \mathcal{A}^{i j}=0
$$

We get the following derived quantities

$$
\begin{aligned}
H_{\mu \nu \rho}^{(0)} & =-\frac{3}{2} l_{[\mu} \mathcal{A}_{\nu \rho]}, \\
\Omega_{+\mu}^{(0) a b} & =\omega_{\mu}^{a b}(e)+H_{\mu}^{(0) a b}=-2 l^{[a} \mathcal{A}_{\mu}^{b]}, \\
\Omega_{-\mu}^{(0) a b} & =\omega_{\mu}^{a b}(e)-H_{\mu}^{(0) a b}=+\mathcal{A}^{a b} l_{\mu}, \\
R_{\mu \nu}^{(0) a b}\left(\Omega_{+}\right) & =-2 l^{[a} \partial^{b]} \mathcal{A}_{\mu \nu}, \\
R_{\mu \nu}^{(0) a b}\left(\Omega_{-}\right) & =-2 l_{[\mu} \partial_{\nu]} \mathcal{A}^{a b} .
\end{aligned}
$$

Observe that the expressions above are true even though we only have related the transverse components of $A$ and $B$ in eq. (40), and we have said nothing about $A_{u}$ and $B_{u}$. The reason is that $B_{u}$ enters in these equations only through $H^{(0)}$, and, actually, it does not contribute to any of the components $H_{\mu \nu \lambda}^{(0)}$ (it is "pure gauge"). This fact offers the possibility of choosing $B_{u}$ so that $\mathcal{A}_{a b}=-\mathcal{B}_{a b}$.

Now let us consider the $\alpha^{\prime}$ corrections. It is well known that in the calculation of the string effective action one has to add to $S^{(0)}$ the Lorentz and Yang-Mills Chern-Simons terms which play a crucial role in the Green-Schwarz anomaly cancellation mechanism. These terms break supersymmetry. To restore supersymmetry order by order in $\alpha^{\prime}$, one has to add to $S^{(0)}$ an infinite series of higher order terms in $\alpha^{\prime}$ ?. By the procedure of adding terms to restore supersymmetry, the $O\left(\alpha^{\prime 3}\right)$ effective action was obtained in [3]

$$
\begin{aligned}
S= & \frac{1}{2} \int d^{10} x \sqrt{-g} e^{-2 \phi}\left(-R+4(\partial \phi)^{2}-\frac{1}{3} H^{2}+\right. \\
& \left.+\frac{1}{2} T+2 \alpha^{\prime} T^{\mu \nu} T_{\mu \nu}+6 \alpha^{\prime} T^{\mu \nu \lambda \rho} T_{\mu \nu \lambda \rho}+O\left(\alpha^{\prime 4}\right)\right),
\end{aligned}
$$

\footnotetext{
6 Another example of an interesting supersymmetric solution with a constant Killing spinor in cartesian coordinates is the purely magnetic extreme dilaton black-hole solution in $d=4$ in stringy metric [2]

${ }^{7}$ The supersymmetrization can be achieved either by the Noether method [17] or by superspace methods (for a recent review of the latter method, see [18] and references therein).
} 
where the antisymmetric tensor $T_{\mu \nu \lambda \rho}$, the symmetric tensor $T_{\mu \nu}$ and the scalar $T$ are given by

$$
\begin{aligned}
T_{\mu \nu \lambda \rho} & =2 \alpha^{\prime}\left(R_{[\mu \nu}{ }^{a b}\left(\Omega_{-}\right) R_{\lambda \rho]}{ }^{a b}\left(\Omega_{-}\right)+\frac{1}{30} \operatorname{tr} F_{[\mu \nu} F_{\lambda \rho]}\right), \\
T_{\mu \nu} & =2 \alpha^{\prime}\left(R_{\mu \lambda}{ }^{a b}\left(\Omega_{-}\right) R_{\nu}^{\lambda}{ }_{\nu}{ }^{a b}\left(\Omega_{-}\right)+\frac{1}{30} \operatorname{tr} F_{\mu \lambda} F_{\nu}^{\lambda}\right), \\
T & =T_{\mu}{ }^{\mu} .
\end{aligned}
$$

In the above expression there are explicit and implicit $\alpha^{\prime}$ corrections. The explicit corrections always appear via $T$-tensors, and they are essentially the $\alpha^{\prime}$ factors in front of eqs. (49)-(51). The implicit $\alpha^{\prime}$ corrections always appear via the torsion $H$ which is defined by the following iterative procedure: At the lowest order $H$ is just $H^{(0)}$, defined in eq. (39) and it is given by eq. (43) for our solutions. With $H^{(0)}$ we calculate the lowest order $\Omega_{ \pm}=\Omega_{ \pm}^{(0)}$ by using its definition eq. (20). $\Omega_{+}$and $\Omega_{-}$are given in eqs. (44) and (45) for our solutions. At first order in $\alpha^{\prime}$, $H=H^{(1)}$ is $H^{(0)}$ corrected by the Yang-Mills Chern-Simons term and the Lorentz Chern-Simons term corresponding to the zero-order $\Omega_{-}=\Omega_{-}^{(0)}$ :

$$
\begin{aligned}
H_{\mu \nu \rho}^{(1)}= & \partial_{[\mu} B_{\nu \rho]}-\frac{1}{5} \alpha^{\prime} \operatorname{tr}\left\{V_{[\mu} \partial_{\nu} V_{\rho]}-\frac{1}{3} V_{[\mu} V_{\nu} V_{\rho]}\right\}- \\
& -6 \alpha^{\prime}\left\{\Omega_{[\mu-}^{(0) a b} \partial_{\nu} \Omega_{\rho]-}^{(0) a b}-\frac{1}{3} \Omega_{[\mu-}^{(0) a b} \Omega_{\nu-}^{(0) a c} \Omega_{\rho-]}^{(0) c b}\right\},
\end{aligned}
$$

With $H^{(1)}$ one would get $\Omega^{(1)}$ using again its definition eq. (20) and $H^{(2)}$ would be given by the above expression but with $\Omega^{(0)}$ replaced by $\Omega^{(1)}$. Iterating this procedure one gets the all-order expression $H$ for the torsion which involves the promised infinite series of corrections. Of course, by this procedure, any tensor containing the torsion (or the torsionful spin connection) receives an infinite number of implicit string corrections. This applies to the $T$-tensors as well.

One may verify that the lowest order Lorentz Chern-Simons term and the Yang-Mills ChernSimons term vanish identically for these solutions. This means that $H^{(1)}=H^{(0)}$, and this in turn implies that $\Omega^{(1)}=\Omega^{(0)}$ etc. The conclusion is that all the implicit string correction vanish for this class of solutions and the expressions in eqs. (43)-(47) are exact to all orders.

Next we have to study the $T$-tensors for these solutions. One may easily establish that the all-order expressions for them are

$$
\begin{aligned}
T_{\mu \nu \lambda \rho} & =T=0, \\
T_{\mu \nu} & =-2 \alpha^{\prime} l_{\mu} l_{\nu}\left\{\left(\partial_{k} \mathcal{A}^{i j}\right)^{2}-\frac{1}{30}\left(\partial_{k} V^{I J}\right)^{2}\right\} .
\end{aligned}
$$

Hence, the squares of all these tensors, which we need to know to calculate the corrections to the on-shell action, vanish for these solutions:

$$
\left(T_{\mu \nu \lambda \rho}\right)^{2}=\left(T_{\mu \nu}\right)^{2}=T^{2}=0 .
$$

Thus the lowest order effective on-shell string action gets no corrections. This is in agreement with the general non-renormalization theorem for the on shell action on bosonic solutions with unbroken supersymmetries, which was presented in [2]. 
Finding the corrections to the equations of motion is more complicated. To study them we first have to vary the action (48) over all the fields present in the theory, and only then substitute the solutions in the corrected equations. It is convenient to start by studying the linear corrections separately. The equations of motion corrected up to first order come from the action

$$
S^{(1)}=\frac{1}{2} \int d^{10} x \sqrt{-g} e^{-2 \phi}\left(-R+4(\partial \phi)^{2}-\frac{1}{3} H^{2}+\frac{1}{2} T\right) .
$$

Note that the notation $S^{(1)}$ implies by definition that all terms of order $\alpha^{\prime 2}$ and higher are neglected. This applies in particular to the implicit $\alpha^{\prime}$ dependence present in $H$ and $T$.

The corrections linear in $\alpha^{\prime}$ to the lowest order equations of motion are derived from the variation $\delta\left(S^{(1)}-S^{(0)}\right) \equiv \delta \Delta S$. It is convenient to perform this variation with respect to the explicit dependence of the action on $g_{\mu \nu}, V_{\mu}, \phi$ and $B_{\mu \nu}$, and then with respect to the implicit dependence on these fields through the torsionful spin connection $\Omega_{-}$, that is

$$
\begin{aligned}
\delta \Delta S= & \frac{\delta \Delta S}{\delta g_{\mu \nu}} \delta g_{\mu \nu}+\frac{\delta \Delta S}{\delta B_{\mu \nu}} \delta B_{\mu \nu}+\frac{\delta \Delta S}{\delta \phi} \delta \phi+ \\
& +\frac{\delta \Delta S}{\delta V_{\mu}} \delta V_{\mu}+\frac{\delta \Delta S}{\delta \Omega_{-\mu} a b} \delta \Omega_{-\mu}^{a b},
\end{aligned}
$$

where

$$
\delta \Omega_{-\mu}^{a b}=\frac{\delta \Omega_{-\mu}^{a b}}{\delta g_{\mu \nu}} \delta g_{\mu \nu}+\frac{\delta \Omega_{-\mu}^{a b}}{\delta B_{\mu \nu}} \delta B_{\mu \nu}+\frac{\delta \Omega_{-\mu}^{a b}}{\delta V_{\mu}} \delta V_{\mu} .
$$

The explicit variations are

$$
\begin{aligned}
\frac{\delta \Delta S}{\delta g_{\mu \nu}}= & -\frac{1}{4} \sqrt{-g} e^{-2 \phi}\left(T^{\mu \nu}-g^{\mu \nu} T\right) \\
\frac{\delta \Delta S}{\delta \phi}= & -\frac{1}{2} \sqrt{-g} e^{-2 \phi} T \\
\frac{\delta \Delta S}{\delta B_{\mu \nu}}= & \frac{1}{3} \partial_{\lambda}\left[\sqrt{-g} e^{-2 \phi}\left(H^{(1) \lambda \mu \nu}-H^{(0) \lambda \mu \nu}\right)\right], \\
\frac{\delta \Delta S}{\delta V_{\mu}}= & \frac{1}{15} \alpha^{\prime}\left\{\partial_{\lambda}\left(\sqrt{-g} e^{-2 \phi} F^{\lambda \mu}\right)+\sqrt{-g} e^{-2 \phi}\left[V_{\lambda}, F^{\lambda \mu}\right]+\right. \\
& \left.+\sqrt{-g} e^{-2 \phi} H^{(0) \mu}{ }_{\lambda \rho} F^{\lambda \rho}-V_{\rho} \partial_{\lambda}\left(\sqrt{-g} e^{-2 \phi} H^{(0) \lambda \mu \rho}\right)\right\} .
\end{aligned}
$$

Clearly, the r.h.s. of the last three equations vanish for these solutions, while the first equation reduces to

$$
\frac{\delta \Delta S}{\delta g_{\mu \nu}}=\frac{1}{2} \alpha^{\prime} e^{-2 \phi} l^{\mu} l^{\nu}\left\{\left(\partial_{k} \mathcal{A}^{i j}\right)^{2}-\frac{1}{30}\left(\partial_{k} V^{I J}\right)^{2}\right\} .
$$

We next consider the implicit variations represented by the last term in eq. (57). In Appendix A we explain a lemma proven in ref. [3] that shows that all these implicit variations are proportional to the lowest order equations of motion of the different fields. We therefore conclude that these solutions of the lowest order equations of motion are solutions also of the equations of motion 
corrected to order $\alpha^{\prime}$ if $T_{\mu \nu}=0$. The latter condition can be fulfilled by embedding the torsionful spin connection in an $S O(8)$ subgroup of the gauge group and identifying

$$
\partial_{k} \mathcal{A}^{i j}=\frac{1}{\sqrt{30}} \partial_{k} V^{I J}
$$

Here the Yang-Mills indices $I J$ refer to the adjoint representation of $S O(8)$. Notice that $S O(8)$ is contained both in $S O(32) / Z_{2}$ and in $E(8) \times E(8)$. In view of their very special properties, we will call the specific class of solutions that satisfy (64) supersymmetric string waves (SSW) . This concludes the discussion of the corrections linear in $\alpha^{\prime}$.

Now let us consider the higher order corrections. The general structure of the bosonic part of the effective action, which can be obtained by the procedure outlined before based upon the restoration of supersymmetry, has been conjectured in ref. [3] to be of the form

$$
\mathcal{L}_{\text {eff }}=\sum_{n=0,1,2, \ldots} \alpha^{\prime n}\left(a_{n} R T^{n}+b_{n} T^{n+1}\right)
$$

where $R$ is the Riemann curvature tensor and $T \equiv \alpha^{\prime}\left(R^{2}+F^{2}\right)$. This has been proven up to order $\alpha^{4}$ in ref. [3]. The terms proportional to $a_{0}$ and $b_{0}$ have just been discussed. It is known that there are no terms proportional to $a_{1}$. The $b_{1}$ terms are given by

$$
\alpha^{\prime} \int d^{10} x \sqrt{-g} e^{-2 \phi}\left(T^{\mu \nu} T_{\mu \nu}+3 T^{\mu \nu \lambda \rho} T_{\mu \nu \lambda \rho}\right)
$$

In the variations of the higher order terms, one should again distinguish between the variations with respect to the explicit dependence on the fields $g_{\mu \nu}, \phi$ and $V_{\mu}$ and the variations with respect to the implicit dependence of these terms on the previous fields and $B_{\mu \nu}$ through the torsionful spin-connection $\Omega_{-}$. It is easy to see that the variations of the first kind do not contribute to the field equations for the SSW. The same applies to the second kind of variations. As an example, we show how this works for the $T^{2}$ terms written above.

Varying with respect to the spin connection and using the fact that $T^{\mu \nu \rho \lambda}=0$ for the SSW, one finds that the only remaining terms are

$$
T^{\mu \nu} R_{\mu}{ }^{\lambda} a b\left(\Omega_{-}\right)\left[D_{\lambda}\left(\Omega_{-}\right) \delta \Omega_{-\nu}^{a b}-D_{\nu}\left(\Omega_{-}\right) \delta \Omega_{-\lambda}^{a b}\right] .
$$

For the SSW solution $T^{\mu \nu} \sim l^{\mu} l^{\nu}$, and

$$
l^{\mu} R_{\mu \nu}^{a b}\left(\Omega_{-}\right)=l_{a} R_{\mu \nu}^{a b}\left(\Omega_{-}\right)=0
$$

Hence, the remaining terms also vanish.

Finally, we consider the corrections to the supersymmetry transformation laws and the Killing spinors. In ref. [3] it was shown that the corrections to order $\alpha^{4}$ are proportional to $T$-tensors and at most one torsionful curvature tensor. Therefore, these corrections vanish for SSW that satisfy the embedding equation (64). Actually, this condition is not necessary in order to establish the vanishing of the corrections to the Killing spinors. The reason is that without the condition (64), the only non-vanishing $T$-tensors are $T_{\mu \nu} \sim l_{\mu} l_{\nu}$. Since our fermionic fields have at most one free index, one of the indices of $T_{\mu \nu}$ has to be contracted either with a curvature tensor or with an 
antisymmetrized gamma matrix $\gamma^{\left[\mu_{1} \ldots \mu_{n}\right]}$. In the first case the correction vanishes due to eq. (68), and in the second case due to the fact that $l_{\mu} \gamma^{\mu} \epsilon=0$. Our Killing spinors, which were obtained in the zero order approximation, satisfy the corrected equations as well.

As we have discussed before, we have only considered the terms that have to be added to the effective action in order to supersymmetrize the Lorentz and Yang-Mills Chern-Simons term. It is well known, however, that other terms occur in the superstring effective action that are not related by supersymmetry to the Lorentz and Yang-Mills Chern-Simons forms, for instance terms of the form $\zeta(3) R^{4}$ [19], 20 Nevertheless, all the terms found so far are at least quartic in the Riemann or Yang-Mills curvature tensors or in the torsion tensor and, therefore, by simple null vector counting, they are harmless.

Our final conclusion is that the on-shell action, the fields that solve the lowest order equations of motion and the Killing spinors for the SSW solutions do not receive any higher order string corrections.

\section{Summary and Conclusion}

In this paper we have found a quite general class of plane wave type solutions of superstring theory, with one half of the space-time supersymmetries unbroken and whose 8 Killing spinors are constant. They are solutions to the field equations of the zero slope limit of the superstring effective action and do not receive any higher order string corrections. The metric, the axion and the gauge fields of these solutions are all described in terms of one vector function of the light-cone coordinate $u$ and of the transverse coordinates $x^{i}$,

$$
A_{\mu}\left(u, x^{i}\right)=\left\{A_{u} \equiv \frac{1}{2} K\left(u, x^{i}\right), A_{v}=0, A_{i}\left(u, x^{i}\right)\right\}, \quad i=1, \ldots, 8 .
$$

They are given by

$$
g_{\mu \nu}=\eta_{\mu \nu}+2 l_{(\mu} A_{\nu)}, \quad H_{\mu \nu \lambda}=3 \partial_{[\mu} l_{\nu} A_{\lambda]}, \quad F_{\mu \nu}^{I J}=2 \sqrt{30} \partial_{[\mu} \mathcal{A}^{i j} l_{\nu]} .
$$

where $\mathcal{A}^{i j}=\partial^{[i} A^{j]}$. The dilaton $\phi(u)$ is a function of $u$ only. The equations that $A_{u}=\frac{1}{2} K\left(u, x^{i}\right)$ and $A_{i}\left(u, x^{i}\right)$ have to satisfy are

$$
\begin{aligned}
& \triangle K+2 \partial^{i} A_{i}^{\prime}+4 \phi^{\prime \prime}=0 \\
& \triangle \mathcal{A}^{i j}=0
\end{aligned}
$$

These solutions are based upon a special conspiracy between the geometry, the axion field and the gauge field. In the non-supersymmetric plane waves considered in [12], [15] there are no relations between the metric, the axion and the gauge fields, besides those coming from the equations of motion. In the zero slope limit the plane waves are supersymmetric if the geometry and the axion field are related by $\partial_{i} \mathcal{A}^{\mu \nu}=-\partial_{i} \mathcal{B}^{\mu \nu}$. The requirement of absence of $\alpha^{\prime}$ corrections to the zero slope supersymmetric solutions puts an even stronger constraint on the solutions: it forces us to

\footnotetext{
${ }^{8}$ The supersymmetrization of the most general $R^{4}$ terms has recently been considered in ref. [21.
} 
embed the torsionful spin connection in the gauge group and the gauge field is also now expressed in terms of the same vector field $A_{\mu}\left(u, x^{i}\right)$. This is possible due to the fact that both $\mathcal{A}^{i j}$ and $V^{I J}$ satisfy the same (harmonic) equations of motion.

Our SSW solutions extend the supersymmetric exact plane wave solutions of string theory studied before by Güven [7] in the following sense. In Güven's solutions, the function $K\left(u, x^{i}\right)$ is quadratic in the $x^{i}$, the non-diagonal functions in the metric $A_{i}\left(u, x^{i}\right) d x^{i} d u$ are absent, and the axion $H_{u i j}$ is a function of $u$ only, i.e. the dependence on $x^{i}$ in $B_{u i}$ is linear in $x^{i}$. There are no relations between the geometry, axion and gauge fields, besides those coming from the equations of motion. Due to the restricted dependence of these solutions on the transverse dimensions, it was not necessary to relate the axion, graviton and gauge fields. Also, there was no need to know the explicit form of the string corrections to prove that they vanish. The existence of a covariantly constant null vector along with the simple dependence on $x^{i}$ was sufficient to establish the non-renormalization theorem.

The SSW, which we have studied, have unconstrained dependence on the transverse coordinates $x^{i}$. The price to pay for making the corrections vanish is the existence of specific relations between different fields in the solution. This means, for example, that generalizations of the Aichelburg-Sexl geometry, where functions like $\ln \left(x_{i} x^{i}\right)$ are present, or solutions where any other complicated dependence on $x^{i}$ appears in a metric of the general form (22), can be investigated in the framework of this SSW. In this paper we have shown that such metrics with the inclusion of appropriate dilaton, axion and gauge fields also belong to the class of perturbatively exact stringy supersymmetric solutions.

It is interesting to compare our SSW solutions with the supersymmetric string solitons (SSS) studied in [1]. The common features are the following. In both cases it is crucial that the supersymmetry transformations depend on $\Omega_{+\mu}^{a b}$ torsionful spin connections, and that the $\alpha^{\prime}$ string corrections depend on $\Omega_{-\mu}{ }^{a b}$. Both for SSW and SSS the embedding of the spin connection into the gauge group is a necessary condition for the absence of higher order string corrections. In addition, the interchange identity between the two types of curvature (31) ensures the consistency of the solutions. In both cases there exists a conspiracy between the metric and the axion field.

A difference, however, is that the dilaton plays a crucial role in the construction of the SSS solution but does not play any particular role in the SSW solution. Furthermore, in the SSS case, the existence of supersymmetry relies on the four-dimensional space-time self-duality and the unbroken supersymmetries are given by chiral spinors with $\epsilon_{+}=0$. In the SSW case, the preserved supersymmetries depend on the existence of the covariantly constant null vector $l_{\mu}$ and the Killing spinors are constant spinors $\epsilon$ such that $l_{\mu} \gamma^{\mu} \epsilon=0$.

Finally, it would be interesting to investigate special examples of our SSW solutions and apply sigma model duality transformations [22 to study how the supersymmetry and non-renormalization properties behave under them. In particular, it has been established before [23] that the metric outside an extremal fundamental string is the dual of a plane fronted wave metric with a dilaton and $A_{u}$-function in the metric, that describes a string boosted to the speed of light. These plane waves have vanishing axion field and vanishing function $A_{i}$ in the metric and no Yang-Mills field. They satisfy our definition of supersymmetric string waves, given in eqs. (70). They are the trivial case in which the conspiracy between metric, axion and gauge field is achieved by the choice 
$A_{i}=0$. It would be interesting to investigate whether the more general supersymmetric string waves studied in our paper generate new interesting geometries via sigma model duality.

On a more fundamental level, one may try to develop a new approach to string quantum gravity starting with supersymmetric string waves. The standard quantum field theory approach is based on the flat-space "plane waves" $\sim e^{i k \cdot x}$, which are solutions of the linearized Einstein equations. The special class of gravitational waves investigated in this paper, which are solutions of the non-linear Einstein equations, and even more, of the non-linear Einstein equations with all $\alpha^{\prime}$-corrections taken into account, may serve as the basis for a new expansion of the path integral for quantum gravity.

\section{Acknowledgements}

The authors wish to thank G. Horowitz for bringing to our attention the issue of the nonrenormalization theorem for gravitational waves. We are grateful to A. Linde and A. Peet for useful discussions.

The work of E. B., R.K. and T.O. was partially supported by a NATO Collaborative Research Grant. The work of E. B. has been made possible by a fellowship of the Royal Netherlands Academy of Arts and Sciences (KNAW). E. B. would like to thank the Physics Department of Stanford University for its hospitality. The work of R.K. was supported in part by NSF grant PHY8612280 and Radway Fellowship in the School of Humanities and Sciences at Stanford University. The work of T.O. was supported by a Spanish Government M.E.C. postdoctoral grant. 


\section{A Lemma}

In this Appendix we give the following Lemma which is used in the text when discussing the string corrections. A proof of (the supersymmetric version of) this Lemma can be found in [3].

Lemma. For arbitrary transformations $\delta \Omega_{\mu-}^{a b}$ the variation of the action

$$
S=\frac{1}{2} \int d^{10} x \sqrt{-g} e^{-2 \phi}\left(-R+4(\partial \phi)^{2}-\frac{1}{3} H^{2}+\frac{1}{2} T\right)
$$

is given by

$$
\delta S=2 \alpha^{\prime} \int d^{10} x\left(\delta_{1} \mathcal{L}+\delta_{2} \mathcal{L}\right)
$$

with

$$
\begin{aligned}
& \delta_{1} \mathcal{L}=6 \mathcal{B}^{\mu \nu} \Omega_{\mu-}^{a b} \delta \Omega_{\nu-}^{a b}+2\left(4 \tilde{\mathcal{E}}_{\rho a}-e_{\rho a} \tilde{\Phi}+6 \tilde{\mathcal{B}}_{\rho a}\right)\left[(\sqrt{-g})^{-1} e^{2 \phi} D_{\lambda}\left(\Omega_{+}\right)\left(\sqrt{-g} e^{-2 \phi} e^{\nu a} \delta \Omega_{\nu-}^{\lambda \rho}\right)\right] \\
& \delta_{2} \mathcal{L}=6 D_{\mu}\left(\Omega_{-}\right)\left(\sqrt{-g} e^{-2 \phi} T^{\mu \nu a b}\right) \delta \Omega_{\nu-}^{a b}+6 \sqrt{-g} e^{-2 \phi} H^{\nu \lambda \rho} T_{\lambda \rho a b} \delta \Omega_{\nu-}^{a b}
\end{aligned}
$$

where $T_{\mu \nu \lambda \rho}$ is defined in (49) and $\tilde{\Phi}, \tilde{\mathcal{E}}_{\mu \nu}$, and $\tilde{\mathcal{B}}_{\mu \nu}$ are given by the expression of $\Phi, \mathcal{E}_{\mu \nu}$, and $\mathcal{B}_{\mu \nu}$, the lowest order equations of motion corresponding to the fields $\phi, B_{\mu \nu}$ and $g_{\mu \nu}$ respectively but where $H^{(0)}$ is replaced by $H$. These lowest order equations are given by

$$
\begin{aligned}
\Phi & \equiv \frac{\delta S^{(0)}}{\delta \phi}=\sqrt{-g} e^{-2 \phi}\left[R-4 \nabla_{\mu} \partial^{\mu} \phi+4(\partial \phi)^{2}+\frac{1}{3}\left(H^{(0)}\right)^{2}\right] \\
\mathcal{E}_{\mu \nu} & \equiv \frac{\delta S^{(0)}}{\delta g^{\mu \nu}}=-\frac{1}{2} \sqrt{-g} e^{-2 \phi}\left(R_{\mu \nu}-2 \nabla_{\mu} \partial_{\nu} \phi+H_{\mu \lambda \rho}^{(0)} H_{\nu}^{(0) \lambda \rho}-g_{\mu \nu} \Phi\right) \\
\mathcal{B}^{\mu \nu} & \equiv \frac{\delta S^{(0)}}{\delta B_{\mu \nu}}=\frac{1}{3} \partial_{\lambda}\left[\sqrt{-g} e^{-2 \phi}\left(H^{(0)}\right)^{\lambda \mu \nu}\right]
\end{aligned}
$$

Note that the combination

$$
-2 \tilde{\mathcal{E}}_{\mu \nu}-3 \tilde{\mathcal{B}}_{\mu \nu}+\frac{1}{2} g_{\mu \nu} \tilde{\Phi}=\sqrt{-g} e^{-2 \phi}\left(R_{\mu \nu}\left(\Omega_{+}\right)-2 \nabla_{\mu}\left(\Gamma_{+}\right) \partial_{\nu} \Phi\right)
$$

is precisely that in which the $H$-dependence can be absorbed into a spin connection with torsion $\Omega_{+}$. 


\section{B Notation and conventions}

We use the notation and conventions of [3]. However, in order to conform as much as possible with the recent literature [1] we have made the following redefinitions with respect to [3]: $\phi \rightarrow$ $e^{2 \phi / 3}, B_{\mu \nu} \rightarrow \frac{1}{3} \sqrt{2} B_{\mu \nu}, A_{\mu} \rightarrow V_{\mu}$ and $\lambda \rightarrow-\frac{1}{4} \sqrt{2} \lambda$. We use a metric with mostly minus signaturef. Our conventions for the Riemann tensor are

$$
R_{\mu \nu \rho}{ }^{\sigma}=\partial_{\mu}\left\{\begin{array}{c}
\sigma \\
\nu \rho
\end{array}\right\}-\partial_{\nu}\left\{\begin{array}{c}
\sigma \\
\mu \rho
\end{array}\right\}+\left\{\begin{array}{c}
\sigma \\
\mu \lambda
\end{array}\right\}\left\{\begin{array}{c}
\lambda \\
\nu \rho
\end{array}\right\}-\left\{\begin{array}{c}
\sigma \\
\nu \lambda
\end{array}\right\}\left\{\begin{array}{c}
\lambda \\
\mu \rho
\end{array}\right\}
$$

where $\left\{\begin{array}{c}\rho \\ \mu \nu\end{array}\right\}$ are the standard Christoffel symbols:

$$
\left\{\begin{array}{c}
\rho \\
\mu \nu
\end{array}\right\}=\frac{1}{2} g^{\rho \lambda}\left\{\partial_{\mu} g_{\nu \lambda}+\partial_{\nu} g_{\mu \lambda}-\partial_{\lambda} g_{\mu \nu}\right\}
$$

The Ricci tensor and the curvature scalar are defined by:

$$
R_{\mu \nu}=g^{\lambda \rho} R_{\mu \lambda \nu \rho}, \quad R=R_{\mu}{ }^{\mu}
$$

The standard general covariant derivative $\nabla_{\mu}$ is

$$
\nabla_{\mu} V_{\nu}=\partial_{\mu} V_{\nu}-\left\{\begin{array}{c}
\rho \\
\mu \nu
\end{array}\right\} V_{\rho}, \quad \nabla_{\mu} V^{\nu}=\partial_{\mu} V^{\nu}+\left\{\begin{array}{c}
\nu \\
\mu \rho
\end{array}\right\} V^{\rho}
$$

The supersymmetry transformation rules (17)-(19) involve the zehnbein fields $e_{\mu}{ }^{a}$ and their inverses $e^{a}{ }_{\mu}$. They are related to the metric tensor and its inverse via

$$
g_{\mu \nu}=e_{\mu}^{a} e_{\nu}^{b} \eta_{a b}, \quad g^{\mu \nu}=e_{a}^{\mu} e_{b}^{\nu} \eta_{a b}
$$

The spin connection field $\omega_{\mu}^{a b}(e)$ is defined in terms of derivatives of the zehnbein fields as follows:

$$
\omega_{\mu}^{a b}(e)=-e^{\nu[a}\left(\partial_{\mu} e_{\nu}^{b]}-\partial_{\nu} e_{\mu}^{b]}\right)-e^{\rho[a} e^{\sigma b]}\left(\partial_{\sigma} e_{c \rho}\right) e_{\mu}^{c}
$$

and it is related to the Christoffel symbols by

$$
\omega_{\mu}^{a b}=-e_{\nu}^{a} e^{\rho b}\left\{\begin{array}{c}
\nu \\
\mu \rho
\end{array}\right\}+e^{\nu b} \partial_{\mu} e_{\nu}^{a}
$$

The curvature tensor corresponding to this spin connection field is defined by

$$
R_{\mu \nu}^{a b}(\omega)=2 \partial_{[\mu} \omega_{\nu]}^{a b}-2 \omega_{[\mu}^{a c} \omega_{\nu] c}^{b}, \quad R(\omega) \equiv e_{a}^{\mu} e_{b}^{\nu} R_{\mu \nu}^{a b}(\omega) .
$$

It is related to the Riemann curvature tensor as follows:

$$
R_{\mu \nu \rho \sigma}=R_{\mu \nu}^{a b}(\omega) e_{a \rho} e_{b \sigma} .
$$

\footnotetext{
${ }^{9}$ Note that in [3] the Pauli metric is used. Here we have converted the results of [3] to correspond to a mostly minus metric.
} 
This relation follows as an integrability condition from the zehnbein postulate

$$
\partial_{\mu} e_{\nu}^{a}-\left\{\begin{array}{c}
\rho \\
\mu \nu
\end{array}\right\} e_{\rho}^{a}-\omega_{\mu}^{a b} e_{\nu b}=0
$$

To specify our Ansatz for the metric, axion and dilaton fields, it is convenient to first write the ten-dimensional coordinates $x^{\mu}$ in a light-cone basis:

$$
x^{\mu}=\left(u, v, x^{i}\right), i=1, \ldots, 8, \quad u=\frac{1}{\sqrt{2}}\left(x_{0}+x_{9}\right), v=\frac{1}{\sqrt{2}}\left(x_{0}-x_{9}\right) .
$$

In this paper, all the indices are raised and lowered with the full ten-dimensional metric $g_{\mu \nu}$. In the case in which the metric is given by eq. (22), the relation between upper and lower indices is

$$
\begin{aligned}
\xi^{u} & =\xi_{v}, \\
\xi^{v} & =\xi_{u}-\left(2 A_{u}+\sum_{i+1}^{8} A_{i}^{2}\right) \xi_{v}+\sum_{i=1}^{8} A_{i} \xi_{i}, \\
\xi^{i} & =A_{i} \xi_{v}-\xi_{i} .
\end{aligned}
$$

Note that if $\xi_{v}=0$, the transverse indices $i$ can be raised and lowered with the flat metric. 


\section{References}

[1] A. Dabholkar, G. Gibbons, J.A. Harvey and F. Ruiz Ruiz, Nucl. Phys. B340 (1990) 33. C.G. Callan Jr., J.A. Harvey and A. Strominger, Nucl. Phys. B359 (1991) 611, Nucl. Phys. B367 (1991) 60.

[2] R.E. Kallosh, A.D. Linde, T.M. Ortí n, A.W. Peet, and A. Van Proeyen. Supersymmetry as a Cosmic Censor, Stanford preprint SU-ITP-92-13, hep-th@xxx/9205027, May 1992, to be published in Phys. Rev. D.

[3] E. Bergshoeff and M. de Roo, Nucl. Phys. B328 (1989) 439.

[4] H. W. Brinkmann, Proc. Natl. Acad. Sci. U. S. 9 (1923) 1.

[5] D. Kramer, H. Stephani, M. MacCallum and E. Herlt, Exact Solutions of Einstein's Field Equations (Cambridge U.P., 1980).

[6] P. Aichelburg and R. Sexl, Gen Relativ. Gravit. 2 (1971) 303.

[7] R. Güven, Phys. Lett. 191B (1987) 265.

[8] G.W. Gibbons and C.M. Hull, Phys. Lett. 109B (1982) 190.

[9] K.P. Tod, Phys. Lett. 121B (1983) 241.

[10] D. Amati and C. Klimčik, Phys. Lett 219B (1988) 443; D. Amati, M. Ciafaloni and Veneziano, Nucl. Phys. B347 (1990) 550, and references therein.

[11] H.J. de Vega and N. Sánchez, Nucl. Phys. B317 (1989) 731; H.J. de Vega, M. Ramón Medrano and N. Sánchez, Nucl. Phys. B374 (1992) 425, and references therein.

[12] G.T. Horowitz and A. R. Steif, Phys. Rev. Lett. 64 (1990) 260; G.T. Horowitz, in: Proceedings of Strings '90, College Station, Texas, March 1990 (World Scientific, 1991) and references therein.

[13] H.W. Brinkmann, Math. Annal. 94, (1925) 119.

[14] R.E. Rudd, Nucl. Phys. B352 (1991) 489.

[15] A.A. Tseytlin, String vacuum backgrounds with covariantly constant null Killing vector and 2d quantum gravity, preprint DAMPT-92-49.

[16] A.H. Chamseddine, Nucl. Phys. B185 (1981) 403; E. Bergshoeff, M. de Roo, B. de Wit and P. van Nieuwenhuizen, Nucl. Phys. B195 (1982) 97; G.F. Chapline and N.S. Manton, Phys. Lett. 120B (1983) 105.

[17] L.J. Romans and N.P. Warner, Nucl. Phys. B273 (1986), 320. S.K. Han, J.K. Kim, I.G. Koh and Y. Tanii, Phys. Rev. D34 (1986) 553.

[18] P. Fré and I. Pesando, in Proceedings of the Conference Strings and Symmetries 1991, Stony Brook, N. Berkovits, H. Itoyama, K. Schoutens, A. Sevrin, W. Siegel, P. van Nieuwenhuizen and J. Yamron eds., (World Scientific, 1992). 
[19] M. Grisaru, A.E.M. van de Ven and D. Zanon, Phys. Lett. 173B (1986) 423; Nucl. Phys. B277 (1986) 389, 409.

[20] D.J. Gross and E. Witten, Nucl. Phys. B291 (1987) 41.

[21] M. de Roo, H. Suelmann and A. Wiedemann, The supersymmetric effective action of the heterotic string in ten dimensions, preprint UG-9/92, hep-th@xxx/9210099.

[22] T. Buscher, Phys. Lett. 194B (1987) 59, Phys. Lett. 201B (1988) 466.

[23] J.H. Horne, G.T. Horowitz and A.R. Steif, Phys. Rev. Lett. 68 (1992) 568. 